\title{
INDUCED CHARGE AND ELECTRIC FIELD IN DENSE MAGNETIZED FERMIONIC MEDIUM AT FINITE TEMPERATURE
}

\author{
E.V. Reznikov* V.V. Skalozub \\ Oles Honchar Dnipro National University, Dnipro, Ukraine \\ e-mail: reznikovev1988@gmail.com
}

The one-photon vertex in presence of strong magnetic field and finite temperature in dense medium is computed, its properties are investigated. Calculations are performed in analytical forms for two cases: at zero temperature and at high temperature. The integral form of the vertex is obtained for a general case. The tensor function is represented as the sum of Feynman's one-loop diagrams. The induced charge dependence on chemical potential, temperature, and strong magnetic field is investigated in detail. The induced potential is calculated for the case of the infinite medium plate.

Keywords: photon vertex, vertex function, dense medium, induced charge, c-parity violation.

Received 12.11.2019; Received in revised form 17.12.2019; Accepted 23.12.2019

\section{Introduction}

The quantum nature of physical vacuum provides phenomena, which can be observed even on a macroscopic scale, such as the Casimir effect [1], light-by-light scattering or Delbrück scattering $[2,3]$. But, much more processes are forbidden in pure vacuum. It concerns those requiring the photon vertices with an odd number of external photon lines. The Furry theorem [4] suppresses such vertices in the vacuum. But in the presence of a medium with sufficiently high chemical potential, its conditions are not satisfied. This happens because of the c-parity violation. In aforementioned circumstances completely new phenomena could arise.

Phenomena occurring in a dense medium with the presence of the chemical potential were studied in $[5,6]$. But in a real dense medium we face the problem of the "purity" of such medium. There can be strong magnetic fields - up to $10^{15} \mathrm{~T}$ - most certainly arisen in the medium during its creation, in addition to chemical potential, and even spontaneous creation of chromomagnetic field is possible [7, 8]. So, in this paper we extend the aforementioned results for the cases of the strong fields and finite temperature in a dense medium.

From all the series of photon vertices with an odd number of external lines, one-photon vertex can provide phenomena, which may significantly change the medium properties by creating a spontaneous induced charge. To consider such cases, we have to calculate the vertex function generated by the chemical potential, taking into account the presence of strong fields and finite temperature.

In this paper, such a program is implemented in the one-loop approximation. The corresponding tensor is calculated using Feynman's diagram with the exact Green function of an internal fermion line in magnetic field and finite temperature presence. Two approximations are considered - high and zero temperature. The exact expressions for the corresponding tensor components are given. Induced charge magnitude dependence on chemical potential, finite temperature, and strong magnetic field is investigated in detail.

\section{One-photon vertex in a dense medium}

The one-photon vertex tensor, which exists in the case of the one-loop approximation for zero temperature, is defined in a dense medium and non-zero magnetic field as the following

$$
\Pi_{v}=\frac{-i e}{(2 \pi)^{3}} \int d^{4} p \operatorname{Tr}\left[\frac{\gamma_{v}(-i \hat{p}+m)}{p^{2}+m^{2}}\right]
$$


where $v$ is an index that runs values from one to four and $G(p)$ is an electron Green's function with the presence of magnetic field and the Euclidean metric is used,

$$
p=\left\{\begin{array}{l}
p_{\rho}, \rho=1,2,3 \\
p_{4}+i \mu, \rho=4
\end{array}, \quad G(p)=\frac{-i p+m}{p^{2}+m^{2}},\right.
$$

$\mu$ is a chemical potential of medium, $\gamma_{\mu}$ are Dirac matrices, and $p_{4}=i p_{0}$.

In case of the stationary and homogeneous field the vertex tensor components are:

$$
\begin{aligned}
& \Pi_{4}=\frac{e}{(2 \pi)^{3}} \int d p_{3} d p_{4} \sum_{n}\left[\frac{p_{4}-i \mu}{\left[\left(p_{4}-i \mu\right)^{2}+(2 n+1) e H-\sigma e H+p_{3}{ }^{2}+m^{2}\right]}\right], \\
& \Pi_{i}=0
\end{aligned}
$$

with spin variable $\sigma= \pm 1$. By summing over $\sigma$ and then over n, we get

$$
\Pi_{4}=\frac{e}{(2 \pi)^{3}} \int d p_{3} d p_{4}\left[\frac{p_{4}-i \mu}{\left[\left(p_{4}-i \mu\right)^{2}+e H+p_{3}{ }^{2}+m^{2}\right]}\right] \text {. }
$$

After integration we obtain

$$
\Pi_{4}=\frac{e}{2 \pi} \Theta\left(\mu^{2}-e H-m^{2}\right)\left(\mu^{2}-e H-m^{2}\right)
$$

where $\Theta\left(\mu^{2}-\left(e H+m^{2}\right)\right)$ is Heaviside's step function.

The structure of the tensor element is similar to that in [5, 6]. But in the presence of a magnetic field the magnitude of the tensor components changes. The function's argument is different from the case of a "pure" medium: the square of the mass is replaced by the sum of the magnetic field strength and the mass squared. In the case under consideration not only the threshold of the function is shifted, but also its value in the allowed regions changes. Thus, at zero temperature and in the presence of strong magnetic field, the induced charge generation is partially or completely suppressed, dependently on the field strength and medium parameters.

\section{One-photon tensor at finite temperature}

Now, using the tensor element (4), we insert the inverse temperature function $\beta=1 / \mathrm{T}$ into it and obtain

$$
\Pi_{4}=\frac{e}{\beta(2 \pi)^{2}} \int d p_{3}\left[\frac{\frac{\pi}{\beta}+i \mu}{\left(\frac{\pi}{\beta}+i \mu\right)^{2}+e H+p_{3}^{2}+m^{2}}-\frac{i \beta \sinh (\beta \mu)}{2\left(\cosh (\beta \mu)+\cosh \left(\beta \sqrt{e H+p_{3}^{2}+m^{2}}\right)\right)}\right] .
$$


In a general case, this integral can be calculated in the form of the sum

$$
\Pi_{4}=\frac{e}{2 \pi} \frac{\left(\frac{\pi}{\beta}+i \mu\right) \Theta(\lambda)}{\beta \sqrt{\left(\frac{\pi}{\beta}+i \mu\right)^{2}+e H+m^{2}}}-\frac{e}{8 \pi^{2}} \int d p_{3} \frac{i \sinh (\beta \mu)}{\left(\cosh (\beta \mu)+\cosh \left(\beta \sqrt{e H+p_{3}{ }^{2}+m^{2}}\right)\right)}
$$

where $\lambda$ is the function of the mass, magnetic field, chemical potential, and $\beta$, $\lambda=\mu^{2}-\frac{\pi^{2}}{\beta^{2}}-m^{2}-e H+\sqrt{\frac{4 \pi^{2} \mu^{2}}{\beta^{2}}+\left(\mu^{2}-\frac{\pi^{2}}{\beta^{2}}-m^{2}-e H\right)^{2}}$. In the low-temperature approximation, it tends to $\lambda=2\left(\mu^{2}-\left(e H+m^{2}\right)\right)$ and $\lambda=0$. Step-function comes from the strict inequality, $\operatorname{Im} \sqrt{\left(\frac{\pi}{\beta}+i \mu\right)^{2}+e H+m^{2}}>0$, So it is defined as $\Theta(\lambda)=\left\{\begin{array}{l}0, \lambda \leq 0, \\ 1, \lambda>0 .\end{array}\right.$

Therefore, in case $\lambda=0$ Heaviside's function equals zero. Then the analytical term tends to the expression $\frac{e}{2 \pi} \frac{i \mu \Theta\left(2\left(\mu^{2}-\left(e H+m^{2}\right)\right)\right)}{\beta \sqrt{e H+m^{2}-\mu^{2}}}$.

The integral can be calculated as the asymptotic at $\beta \rightarrow \infty$. In this case large $p_{3}$ gives a leading contribution. So, we expand the integrand in series in $\left(e H+\mathrm{m}^{2}\right)$. After calculations we obtain

$$
\begin{aligned}
& \frac{e}{8 \pi^{2}} \int d p_{3} \frac{i \sinh (\beta \mu)}{\left(\cosh (\beta \mu)+\cosh \left(\beta \sqrt{e H+p_{3}{ }^{2}+m^{2}}\right)\right)}= \\
& =\frac{e}{2 \pi} \Theta\left(2\left(\mu^{2}-\left(e H+m^{2}\right)\right)\right)\left(\mu^{2}-\left(e H+m^{2}\right)\right)
\end{aligned}
$$

that coincides with (5).

The analytical part of (7) has the linear dependence on $T$ at any temperatures. In the case of low temperature, the imaginary part is greater that the real one. It indicates the existence of the instability in such a state. This can lead to dissipation processes, such as creation of plasmons or generation of transversal photons. The expression (7) displays that the impact of the temperature on processes of the induced charge generation is significant and cannot be reduced to correction factors.

\section{One-photon tensor at high temperature}

In the case of high temperature asymptotic, $\beta$ approaches to zero, hyperbolic sinuses can be replaced by their arguments and cosines tend to one. Then integration of nonanalytic part of (7) can be fully performed and yields

$$
\int d p_{3} F=\frac{i \mu \pi \Theta(\lambda)}{\beta \sqrt{4+\beta^{2}\left(e H+m^{2}\right)}} .
$$


Combining this result and the analytical part of (7), we obtain the exact expression for the temporal element.

$$
\Pi_{4}=\frac{e}{2 \pi} \frac{\left(\frac{\pi}{\beta}+i \mu\right) \Theta(\lambda)}{\beta \sqrt{\left(\frac{\pi}{\beta}+i \mu\right)^{2}+e H+m^{2}}}-\frac{e}{4 \pi} \frac{i \mu \Theta(\lambda)}{\beta \sqrt{4+\beta^{2}\left(e H+m^{2}\right)}}
$$

In the case of $T>>\mu$ this is simplified to

$$
\Pi_{4}=\frac{e}{2 \pi} \Theta(\lambda)\left(\frac{1}{\beta}-\frac{i \mu}{4}\right)
$$

In high temperature approximation, $\lambda$ tends to $\lambda=2 \mu^{2}\left(1-\frac{\left(e H+m^{2}\right) \beta^{2}}{\pi^{2}}\right)$.

Therefore, the contribution of the sum of squared masses is strongly diminished, and the allowed region for the tensor component is any non-zero $\mu$. Thus, we have a linear dependence on the temperature with the addition of a small imaginary correction. The influence of high temperature ensures the generation of the induced charge even for small values of the chemical potential.

\section{Behavior of induced charge and potential}

Aforementioned results were obtained in our previous paper [9]. We used them here to obtain the density of the induced charge. In the low temperature approximation, we get

$$
\begin{aligned}
& \rho(\mu, m, \beta)=2 e \Theta(\lambda) \operatorname{Re}\left(\frac{\left(\frac{\pi}{\beta}+i \mu\right)}{\beta \sqrt{\left(\frac{\pi}{\beta}+i \mu\right)^{2}+e H+m^{2}}}-\frac{e H-\mu^{2}+m^{2}}{1+\cosh \left(\pi \beta \sqrt{e H-\mu^{2}+m^{2}}\right)^{2}}\right), \\
& \lambda=\mu^{2}-e H-m^{2}+\sqrt{\left(e H-\mu^{2}+m^{2}\right)^{2}}+\frac{2 \mu^{2} \pi^{2}}{\left(\mu^{2}-e H-m^{2}\right) \beta^{2}}
\end{aligned}
$$

and in high temperature approximation, we obtain

$$
\begin{aligned}
& \rho(\mu, m, \beta)=2 e \operatorname{Re} \Theta(\lambda)\left(\frac{1}{\beta}-\frac{i \mu}{4}\right) . \\
& \lambda=2 \mu^{2}\left(1-\frac{\left(e H+m^{2}\right) \beta^{2}}{\pi^{2}}\right)
\end{aligned}
$$

We plotted the dependence of induced charge density on temperature for several values of chemical potential and magnetic field.

In the case when the sum of the squared fermion mass and the magnetic field strength is lower than the square of the chemical potential, the approximation of the low temperature gives a substantial density for the induced charge in the field of applicability. But its contribution decreases rapidly at a temperature increase. In the opposite case, the 
low temperature approximation gives zero for the induced charge density and the high temperature approximation has a threshold. At some temperature, dependent on chemical potential and magnetic field, induced charge density occurs sharply. Thus, by changing the magnetic field, we can control at what temperature the induced charge generation begins.
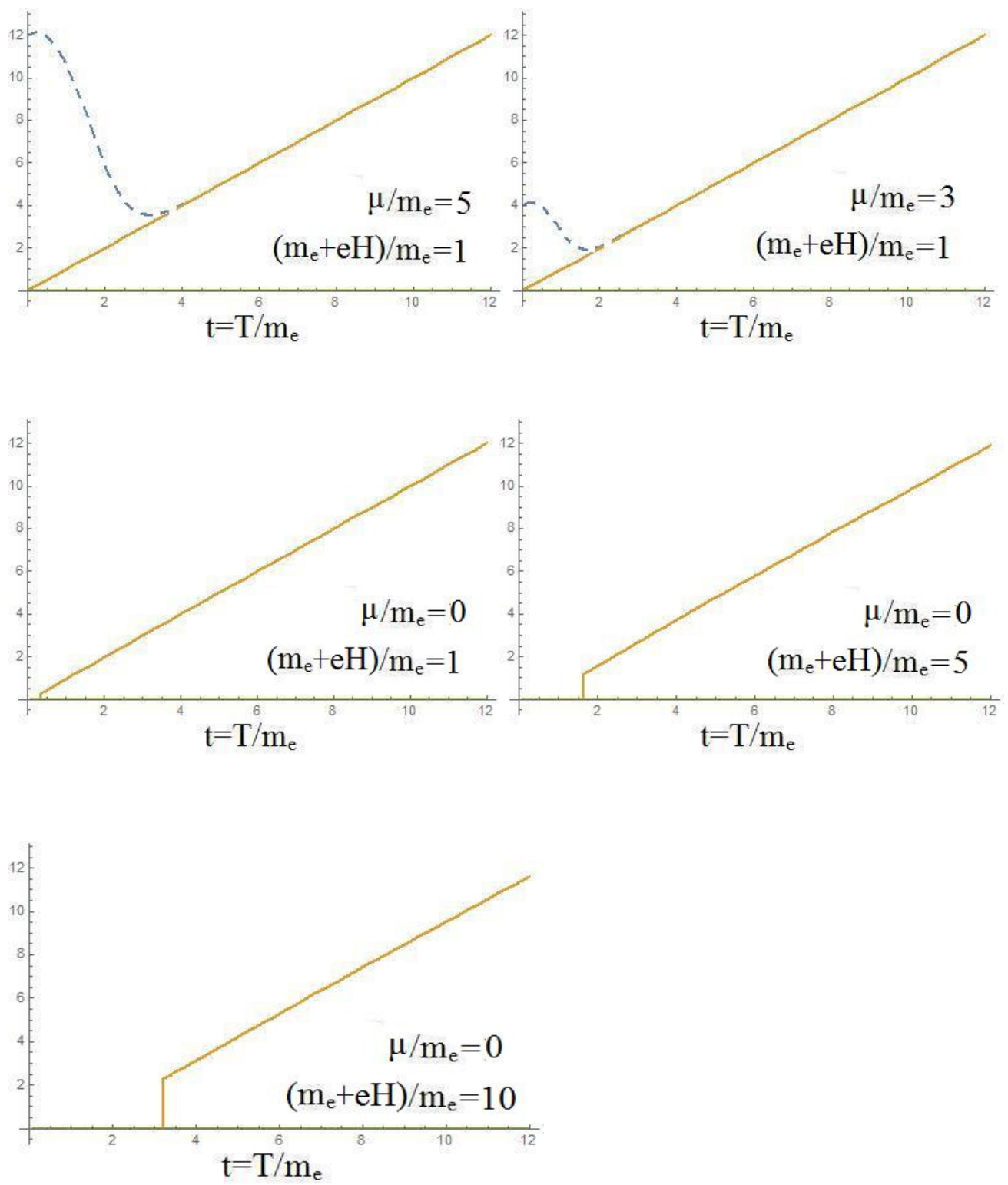

Fig. 1. The temperature dependence of the induced charge density.

The solid line presents the plot for high temperature approximation; the dashed line shows the plot for low temperature approximation. 
We consider the medium confined in the plate of the size $L$ in z-axis direction and infinite in $\mathrm{x}$ and $\mathrm{y}$ directions. The classical potential $\varphi$ is calculated from the equation

$$
\left[\frac{\partial^{2}}{\partial x_{\mu}{ }^{2}}-m_{D}^{2}\right] \varphi=-\rho(\mu, m, \beta)
$$

Making Fourier's transformation to momentum $k$-space, we derive the spectrum of modes $-k_{4}^{2}=k_{x}^{2}+k_{y}^{2}+k_{z}^{2}+m_{D}^{2}$, where $k_{z}^{2}=\left(\frac{2 \pi}{L}\right)^{2} l^{2}, \quad l=0, \pm 1, \pm 2, \ldots$, and $m_{D}^{2}$ is Debye's plasmon mass. The discreteness of $k_{z}$ is due to the periodic boundary condition for the plate: $\varphi(z)=\varphi(z+L)$. The general solution to Eq. (14) is

$$
\varphi\left(x_{4}, x\right)=d+a e^{-i\left(k_{4} x_{4}-k x\right)}+b e^{i\left(k_{4} x_{4}-k x\right)} .
$$

In the case of the zero induced charge $d=0$ and we have two plasmon modes. In presence of induced charge, we use the boundary condition

$$
\varphi\left(\frac{L}{2}\right)=\varphi\left(-\frac{L}{2}\right)=-\frac{\rho(\mu, m, \beta) L^{2}}{2}
$$

that results in the expression

$$
\varphi(z)=\frac{\rho(\mu, m, \beta)}{m_{D}^{2}}\left[1-\frac{1-L^{2} m_{D}^{2} / 2}{\cos \left(k_{z} L / 2\right)} \cos \left(k_{z} z\right)\right] .
$$

The generated potential depends on z-coordinate only.

In case of high temperature Debay's temperature mass has the order $m_{D}^{2} \approx e^{2} T^{2}$. We use this approximation and the explicit form of $\rho(\mu, m, \beta)$ and obtain the approximate expression for the induced potential inside the plate at high temperature,

$$
\varphi(z) \cong \frac{2 \beta}{e^{2}} \Theta\left(2 \mu^{2}\left(1-\frac{\left(e H+m^{2}\right) \beta^{2}}{\pi^{2}}\right)\right)\left[1-\frac{\beta^{2}-L^{2} e^{2} / 2}{\beta^{2} \cos \left(k_{z} L / 2\right)} \cos \left(k_{z} z\right)\right] .
$$

We plotted the dependence of the induced potential of the plate on the z-distance for several values of temperature.

In this plot, we observe the growth of the induced potential in each point of space at temperature increase. The temperature step also exists. Below it the induced potential equals zero. 


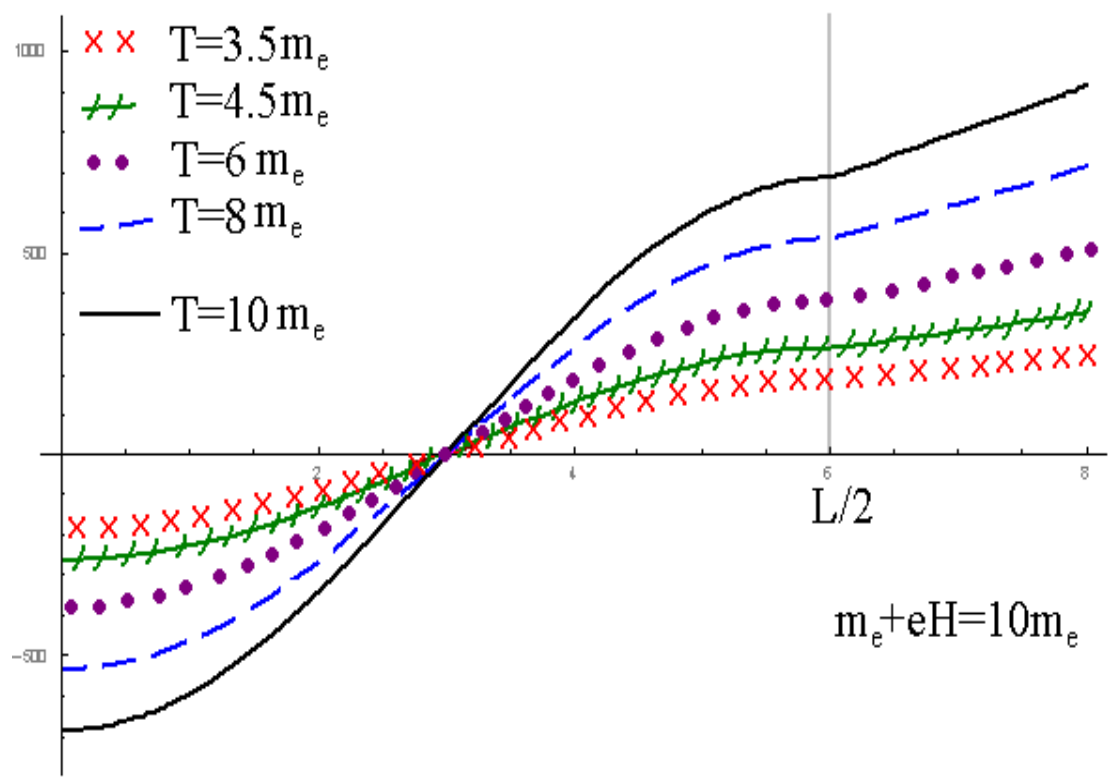

Fig. 2. The dependence of induced potential on distance.

Under the used conditions, the potential at $T \leq 3 m_{e}$ is equal to zero.

\section{Discussion}

The generation of the induced charge in dense medium takes place at sufficiently high value of chemical potential. At the same time, strong electric fields will appear in real dense medium. They could significantly affect the processes that take place in this case.

In $[5,6]$, in particular, the exact expression for the temporal element of the onephoton vertex tensor in dense medium was obtained. This expression determines the induced charge density function. In the present paper, we calculate the tensor element in magnetic field and finite temperature.

Calculations show that the presence of magnetic field in dense medium at zero temperature suppresses the induced charge by adding the magnetic field strength to the mass square in the function argument. Also, the temporal component of the one-photon vertex is obtained in an analytical form in the high temperature approximation. We show that the tensor element - and the density of the induced charge - is proportional to the medium temperature. This important fact means that high temperatures stimulate the charging of media. At the same time, the instabilities are suppressed and the medium is strongly charged. It could result in new effects. We also detect that at sufficiently high temperature the charging starts at not dense media, $\mu \cong 0$. In this case, the magnetic field strength determines the required temperature at which the induced charge arises.

The charging of dense medium is an important phenomenon affecting different processes. The presence of a charge can change the scattering parameters of the particles and cause the creation of new particles - primarily wide variety of photons, but with a sufficiently strong charge - massive particles, too. Moreover, the presence of the induced charge will be an observable explicit signal of the dense medium appearance. Magnetic field strength sets the temperature of the medium, at which charging takes place. The strong spatially alternating potential is generated in the plate at sufficiently high temperature. 


\section{Acknowledgement}

One of the authors, Evgeniy Reznikov, acknowledges the receipt of the grant from the Abdus Salam International Centre for Theoretical Physics, Trieste, Italy.

\section{References}

1. Bordag, M. Advances in the Casimir Effect / M. Bordag, G.L. Klimchitskaya, U. Mohideen, and V.M. Mostepanenko. - Oxford: Oxford Univ. Press, 2009, 749 P.

2. Jaffe, R.L. Casimir effect and the quantum vacuum // Phys. Rev. D. - 2005. Vol. 72, 021301.

3. Grib, A.A. Vakuumnyye kvantoviye effekty v silnykh polyakh / A.A. Grib, S.G. Mamayev, V.M. Mostepanenko. - Moscow: Energoatomizdat, 1988. P. 288.

4. Furry, W. A Symmetry Theorem in the Positron Theory // Phys. Rev. - 1937. Vol. 51. - P. $125-129$.

5. Skalozub, V.V. The polarization operator and the three-photon vertex in QED_2+1 in a dense medium / V.V. Skalozub, A.Yu. Tishchenko // Zh. Eksp. Teor. Fiz. - 1993. - Vol. 104. - P. 3921 - 3927.

6. Skalozub, V.V. Effective Lagrangian and the nonlinear interaction of a nonuniform electromagnetic field in QED 2+1 in a dense fermion medium / V.V. Skalozub, A.Yu. Tishchenko // Journal of Experimental and Theoretical Physics. - 1995. - Vol. 81. - P. 207 - 209.

7. Skalozub, V.V. On magnetization of quark-gluon plasma at the LHC experiment energies / V.V. Skalozub, P.E. Minaiev // Visnyk Dnipropetrovs`koho Universytetu. Seriya: Fizyka. Radioelektronika. - 2016. - Vol. 24. P. 25 - 30.

8. Skalozub, V.V. Magnetized quark-gluon plasma at the LHC / V.V. Skalozub, P.E. Minaiev // Physics of Particles and Nuclei. - 2017. - Vol. 15. - P. 568 - 575.

9. Reznikov, E.V. Induced Charge in Dense Magnetized Fermionic Medium at Finite Temperature / E.V. Reznikov, V.V. Skalozub // Nonlinear Phenomena in Complex Systems. - 2019. - Vol. 22, No. 3. - P. 305 - 309. 